\title{
A novel case of myxoid variant of adrenocortical carcinoma in a patient with multiple endocrine neoplasia type 1
}

\author{
Ko Harada ${ }^{1)}$, Miho Yasuda ${ }^{1)}$, Kou Hasegawa ${ }^{1)}$, Yuto Yamazaki ${ }^{2)}$, Hironobu Sasano $^{2)}$ and Fumio Otsuka ${ }^{1)}$ \\ 1) Department of General Medicine, Okayama University Graduate School of Medicine, Dentistry and Pharmaceutical Sciences, \\ Okayama 700-8558, Japan \\ 2) Department of Anatomic Pathology, Tohoku University Graduate School of Medicine, Miyagi 980-8575, Japan
}

\begin{abstract}
Adrenocortical carcinoma (ACC) is a rare malignancy arising from adrenocortical parenchymal cells. Myxoid ACC is one of the newly identified, rare, but important histological variants of ACC, characterized by the presence of abundant extracellular Alcian Blue-positive myxoid material. Multiple endocrine neoplasia type 1 (MEN1) is an autosomal dominant cancer predisposition syndrome, and the incidence of ACC in MEN1 patients has been reported to be between 1.4\% and $6 \%$. Here, we report the case of a 68-year-old Japanese woman harboring the past history of MEN1 associated with insulinoma, pituitary tumor, and hyperparathyroidism. She presented to our hospital with hypertension and hypokalemia. Imaging studies revealed a right adrenal tumor, and histological examination revealed myxoid ACC. Despite surgical resection of the tumor and mitotane therapy, the patient died 6 months after the surgery. To the best of our knowledge, this is the first reported case of the myxoid variant of ACC in a patient with MEN1. The patient's clinical course was characterized by the development of both multiple endocrine and non-endocrine neoplasm, hyperaldosteronism, and aggressive biological behavior. This case confirmed that myxoid morphology was also associated with aggressive behavior in ACC, but further studies are required to clarify the association between MEN1 and myxoid ACC.
\end{abstract}

Key words: Adrenocortical carcinoma, Multiple endocrine neoplasia type 1, Myxoid variant

\begin{abstract}
ADRENOCORTICAL CARCINOMA (ACC) is a rare malignant neoplasm arising from adrenocortical parenchymal cells [1]. The incidence of ACC is approximately one to two per million population per year $[2,3]$. It usually occurs in adults, with a peak incidence in the fifth decade of life, and women are more likely to be affected than men [4]. Patients with ACC generally have an unfavorable clinical outcome, and the average duration of overall survival has been reported 14.5 months, with a 5 -year mortality rate of $75 \%$ to $90 \%[5,6]$. ACC has a
\end{abstract}

Submitted Feb. 16, 2019; Accepted Apr. 18, 2019 as EJ19-0067 Released online in J-STAGE as advance publication May 22, 2019 Correspondence to: Ko Harada, M.D., Department of General Medicine, Okayama University Graduate School of Medicine, Dentistry and Pharmaceutical Sciences, 2-5-1 Shikata-cho, Kitaku, Okayama 700-8558, Japan.

E-mail:me422084@s.okayama-u.ac.jp

Abbreviations: ACC, Adrenocortical carcinoma; MEN1, Multiple endocrine neoplasia type 1; CT, Computed tomography; SUV max, Maximum standardized uptake value; MRI, Magnetic resonance imaging; SF-1, Steroidogenic factor; 3 $\beta \mathrm{HSD}, 3 \beta$-hydroxysteroid dehydrogenase; P450c17, 17 $\alpha$-hydroxylase; CYP11B1, 11ßhydroxylase; CYP11B2, Aldosterone synthase; DHEA-ST, Dehydroepiandrosterone sulfotransferase heterogenous morphology in its classical form and includes several rare histological variants, including oncocytic, myxoid, and sarcomatoid [7]. Among those subtypes, myxoid variant of ACC was recently identified and characterized by tumor cells arranged in cords, clusters, or pseudoglandular structures that are embedded in a prominent myxoid stromal background. Myxoid ACC is also considered to harbor more aggressive clinical course than non-myxoid ACC $[8,9]$.

Multiple endocrine neoplasia type 1 (MEN1) is an autosomal dominant cancer predisposition syndrome caused by mutations in the MEN1 gene [10, 11]. Among these concurring pathology, adrenal involvement in MEN1 patients is relatively common but most of the lesions are hyperplasia and nonfunctioning [12]. Here, we report the rare case of a patient with MEN1 who developed myxoid ACC during the clinical follow-up.

\section{Case Report}

A 68-year-old Japanese woman with a history of MEN1 presented to our hospital with hypertension and hypokalemia. Her family history was significant in that 
her father had pituitary adenoma and pancreatic cancer, although neither her mother nor her children had any cancer or endocrine diseases. At the age of 38 years, she underwent surgery for resection of the insulinoma but a residual lesion was present in the tail of her pancreas, for which she had been administered diazoxide. At the age of 46 , she received a right total mastectomy for breast cancer. At the age of 47 , a nonfunctional pituitary tumor was detected and transsphenoidal surgery was performed. Subsequently, she underwent transsphenoidal surgeries for recurrent tumors at the ages of 56 and 59. At the age of 57, a parathyroidectomy was performed for primary hyperparathyroidism. At the age of 59, a thymectomy for a thymoma was performed. At the age of 67, she underwent a left partial mastectomy for left breast cancer. Based on this medical history, she was diagnosed with MEN1 associated with insulinoma, pituitary tumors, and hyperparathyroidism, even though MEN1 gene analysis had not been performed.

As part of regular follow ups with our department, the patient received laboratory tests and imaging studies to check for disease recurrence. At the age of 68 , she noticed hypertension and, therefore, visited our hospital. She had lost $10 \mathrm{~kg}$ of weight over the past year. On examination, her blood pressure was elevated to $173 / 112$ $\mathrm{mmHg}$. She did not have any features suggestive of Cushing's syndrome. Her laboratory data were summarized in Table 1. Her serum potassium level was extremely low, at $2.5 \mathrm{mmol} / \mathrm{L}$. Her plasma aldosterone and serum cortisol levels were elevated to $694 \mathrm{pg} / \mathrm{mL}$ and $19.5 \mu \mathrm{g} / \mathrm{dL}$, respectively, while basal ACTH level was low at $1.9 \mathrm{pg} / \mathrm{mL}$. A low-dose dexamethasone (1 $\mathrm{mg}$ ) suppression test revealed a serum cortisol level of $20.0 \mu \mathrm{g} / \mathrm{dL}$. These laboratory data indicated the overproduction of aldosterone and cortisol. Fig. 1 shows the changes in laboratory data and right adrenal tumor over the last 3 years. Laboratory data on presentation showed high blood pressure, increased level of cortisol and aldosterone, and decreased level of potassium, adrenocorticotropin and plasma renin activity, compared to the values at last visit. Computed tomography (CT) showed gradual enlargement of the adrenal tumor over 3 years, which especially rapidly grew in the last 1 year. In $\mathrm{X}-1$ year, we recommended her to receive further evaluation for the right adrenal tumor, but the patient refused it because of personal reasons. CT showed a right adrenal tumor measuring $70 \mathrm{~mm}$ in greatest diameter with 32 Hounsfield units (HU), and contrast-enhanced CT revealed a heterogeneously enhanced adrenal tumor (Fig. 2A-2B). Positron emission tomography/CT showed 18Ffludeoxyglucose uptake in the same region (standardized uptake value [SUV max]: 29.1) (Fig. 2C). Magnetic resonance imaging (MRI) chemical shift imaging revealed a large irregular lesion that did not exhibit signal loss between in-phase and out-of-phase images (Fig. 2D-2E). On T2-weighted imaging, the tumor was found to be heterogeneous and hyperintense to the liver parenchyma (Fig. 2F).

Based on imaging findings and laboratory data, ACC was strongly suspected. At this point, no apparent metastasis to the other organs was detected. She underwent surgical resection of the right adrenal tumor and the right kidney, together with a part of the inferior vena cava. At the same time, a distal pancreatectomy was performed for the residual insulinoma. The adrenal tumor submitted for pathological examination consisted of a solitary darkbrown tumor with a thin fibrous capsule, and had a largest diameter measuring $70 \mathrm{~mm}$ (Fig. 3A). Microscopically, the tumor presented a total Weiss score of 7 out of 9 points, including high nuclear grade, high mitotic rate (24 mitoses per 10 high power fields), eosinophilic cytoplasm, diffuse architecture, venous invasion, sinusoidal invasion, and capsular invasion (Fig. 3B-3D). Alcian blue periodic acid-Schiff (AB-PAS) staining did reveal the presence of the myxoid material (Fig. 3E). Ki-67 labeling index was $21 \%$ in the hot spots (Fig. $3 F$ ). The tumor cells were immunohistochemically positive for steroidogenic factor 1 (SF-1) (Fig. 3G). In addition, 3 $\beta$ hydroxysteroid dehydrogenase (3 $\beta \mathrm{HSD}), 17 \alpha$-hydroxylase (P450c17), 11 $\beta$-hydroxylase (CYP11B1), aldosterone synthase (CYP11B2), and dehydroepiandrosterone sulfotransferase (DHEA-ST) (Fig. 3H-3L). Immunohistochemical study of adjacent normal adrenocortical tissue also revealed decreased expression of DHEA-ST, indicating the suppression of the hypothalamic-pituitaryadrenal axis due to overproduction of cortisol from the ACC.

Based on these pathological and immunohistochemical findings, we finally reached a diagnosis of a myxoid variant of ACC. The patient was started on mitotane therapy at 2 weeks after surgery. However, multiple metastases to her liver were detected at 14 weeks after surgery. The amount of mitotane used at this point was $70 \mathrm{~g}$. The patient died at 24 weeks after the surgery because of hepatic and renal failures.

\section{Discussion}

The patient in our present study was diagnosed with MEN1 associated with insulinoma, pituitary tumor, and hyperparathyroidism. During the clinical course of MEN1, she developed ACC, which was surgically resected. To the best of our knowledge, this is the first reported case of a myxoid variant of ACC associated with MEN1.

MEN1 is an autosomal dominant hereditary syndrome. 
Table 1 Laboratory data

\begin{tabular}{|lr|}
\hline \multicolumn{2}{|c|}{ Complete blood count } \\
White blood cell & $\mathbf{1 1 , 5 1 0} / \mu \mathrm{L}$ \\
Neutrophil & $84.5 \%$ \\
Lymphocyte & $8.3 \%$ \\
Eosinophil & $\mathbf{0 . 1} \%$ \\
Red blood cell & $424 \times 10^{4} / \mu \mathrm{L}$ \\
Hemoglobin & $13.6 \mathrm{~g} / \mathrm{dL}$ \\
Platelet & $39 \times 10^{4} / \mu \mathrm{L}$ \\
& \\
& \\
Tumor marker & $239 \mathrm{U} / \mathrm{mL}$ \\
Soluble interleukin-2 receptor & $3.4 \mathrm{ng} / \mathrm{mL}$ \\
Cytokeratin 19 fragment & $3.05 \mathrm{ng} / \mathrm{mL}$ \\
Carcinoembryonic antigen & $\mathbf{2 4 . 3} \mathrm{ng} / \mathrm{mL}$ \\
Neuron specific enolase & $28.7 \mathrm{U} / \mathrm{mL}$ \\
Carbohydrate antigen 19-9 & \\
& \\
& \\
\hline
\end{tabular}

Biochemistry
Total protein
Albumin
Total bilirubin
Aspartate transaminase
Alanine transaminase
$\gamma$-glutamyl transpeptidase
Lactate dehydrogenase
Sodium

Potassium

Chloride

Corrected calcium

Phosphate

Intact parathyroid hormone $304 \mathrm{pg} / \mathrm{mL}$

Blood urea nitrogen

Creatinine

Fasting plasma glucose

Hemoglobin A1c
$6.1 \mathrm{~g} / \mathrm{dL}$

$3.4 \mathrm{~g} / \mathrm{dL}$

$0.57 \mathrm{mg} / \mathrm{dL}$

$36 \mathrm{U} / \mathrm{L}$

$58 \mathrm{U} / \mathrm{L}$

$96 \mathrm{U} / \mathrm{L}$

$498 \mathrm{U} / \mathrm{L}$

$144 \mathrm{mmol} / \mathrm{L}$

$2.5 \mathrm{mmol} / \mathrm{L}$

$94 \mathrm{mmol} / \mathrm{L}$

$10.7 \mathrm{mg} / \mathrm{dL}$

$2.9 \mathrm{mg} / \mathrm{dL}$

$26.4 \mathrm{mg} / \mathrm{dL}$

$0.99 \mathrm{mg} / \mathrm{dL}$

$112 \mathrm{mg} / \mathrm{dL}$

$5.9 \%$

\begin{tabular}{|lr|}
\hline \multicolumn{2}{|c|}{ Endocrine } \\
Free thyroxine & $1.49 \mathrm{ng} / \mathrm{dL}$ \\
Thyroid stimulating hormone & $0.84 \mu \mathrm{U} / \mathrm{mL}$ \\
Luteinizing hormone & $\mathbf{0 . 5} \mathrm{mIU} / \mathrm{mL}$ \\
Follicle stimulating hormone & $\mathbf{5 . 5} \mathrm{mIU} / \mathrm{mL}$ \\
Testosterone & $13.9 \mathrm{ng} / \mathrm{dL}$ \\
Prolactin & $\mathbf{4 1 . 5} \mathrm{ng} / \mathrm{mL}$ \\
Growth hormone & $0.36 \mathrm{ng} / \mathrm{mL}$ \\
Insulin-like growth factor-1 & $121 \mathrm{ng} / \mathrm{mL}$ \\
Adrenocorticotropin & $\mathbf{1 . 9} \mathrm{pg} / \mathrm{mL}$ \\
Cortisol & $\mathbf{1 9 . 5} \mu \mathrm{g} / \mathrm{dL}$ \\
Plasma renin activity & $\mathbf{0 . 8} \mathrm{ng} / \mathrm{mL} / \mathrm{h}$ \\
Aldosterone & $\mathbf{6 9 4} \mathrm{pg} / \mathrm{mL}$ \\
Dehydroepiandrosterone sulfate & $67 \mu \mathrm{g} / \mathrm{dL}$ \\
Urine free cortisol & $\mathbf{2 4 7 . 8} \mu \mathrm{g} / \mathrm{day}$ \\
Urine aldosterone & $\mathbf{5 8 . 8} \mu \mathrm{g} / \mathrm{day}$ \\
& \\
\hline
\end{tabular}

Abnormal data are shown in bold.

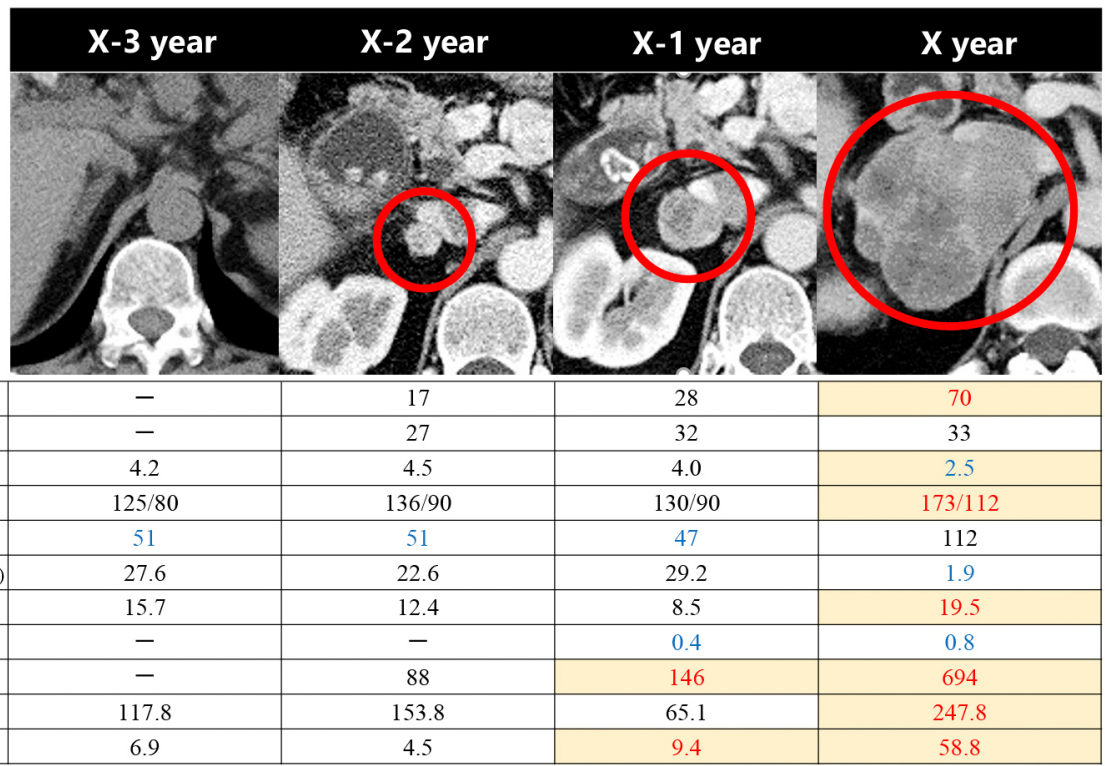

Fig. 1 Progression of tumor size and the related clinical features. Computed tomography (CT) shows gradual enlargement of the adrenal tumor over 3 years, with especially rapid growth in the last 1 year.

The most frequent features of MEN1 cases are primary hyperparathyroidism, pancreatic endocrine tumors, and pituitary adenomas. An involvement of the adrenal gland has been reported in between $20 \%$ and $40 \%$ of patients with MEN1. The incidence of ACC in patients with MEN1 has been reported as being between $1.4 \%$ and $6 \%$
$[12,13]$. The prevalence of ACC has been also reported approximately 10 times higher in patients who have adrenal tumors and MEN1 than in patients who have adrenal incidentalomas without MEN1 [13]. The genetic defects such as $p 53$ and MEN1 mutations are related to the development of ACC [14]. 


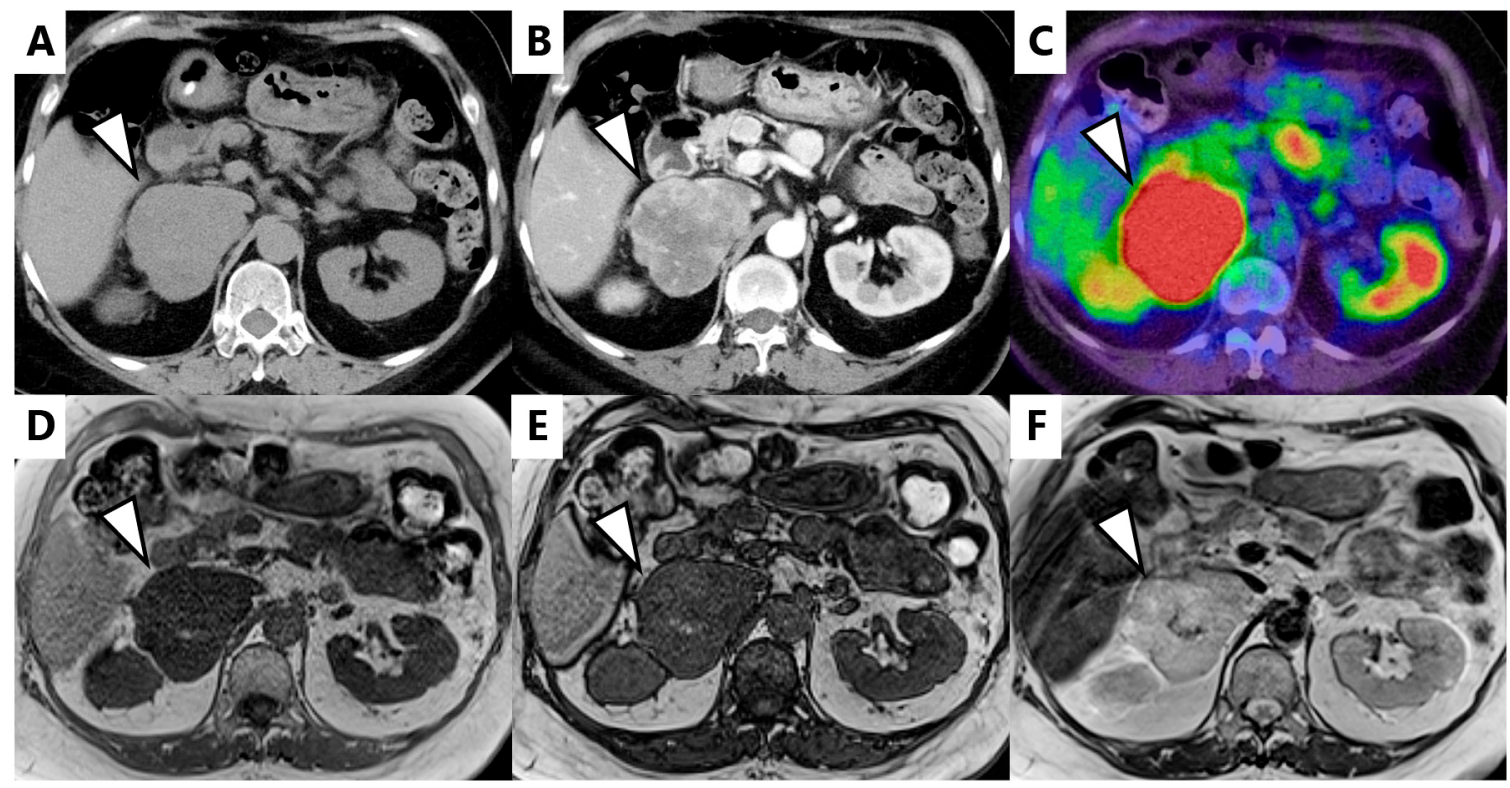

Fig. 2 Imaging findings. Computed tomography (CT) shows a right adrenal tumor $70 \mathrm{~mm}$ in diameter (A, arrowhead), and contrastenhanced CT reveals a heterogeneously enhanced adrenal tumor (B, arrowhead). Positron emission tomography/CT shows 18Ffludeoxyglucose uptake in the same region (standardized uptake value [SUV max]: 29.1) (C, arrowhead). On magnetic resonance imaging (MRI), chemical shift imaging shows a large irregular right-sided lesion that did not exhibit signal loss between in-phase (D, arrowhead) and out-of-phase (E, arrowhead) images. On T2-weighted imaging, the tumor was found to be heterogeneous and hyperintense to the liver parenchyma (F, arrowhead).

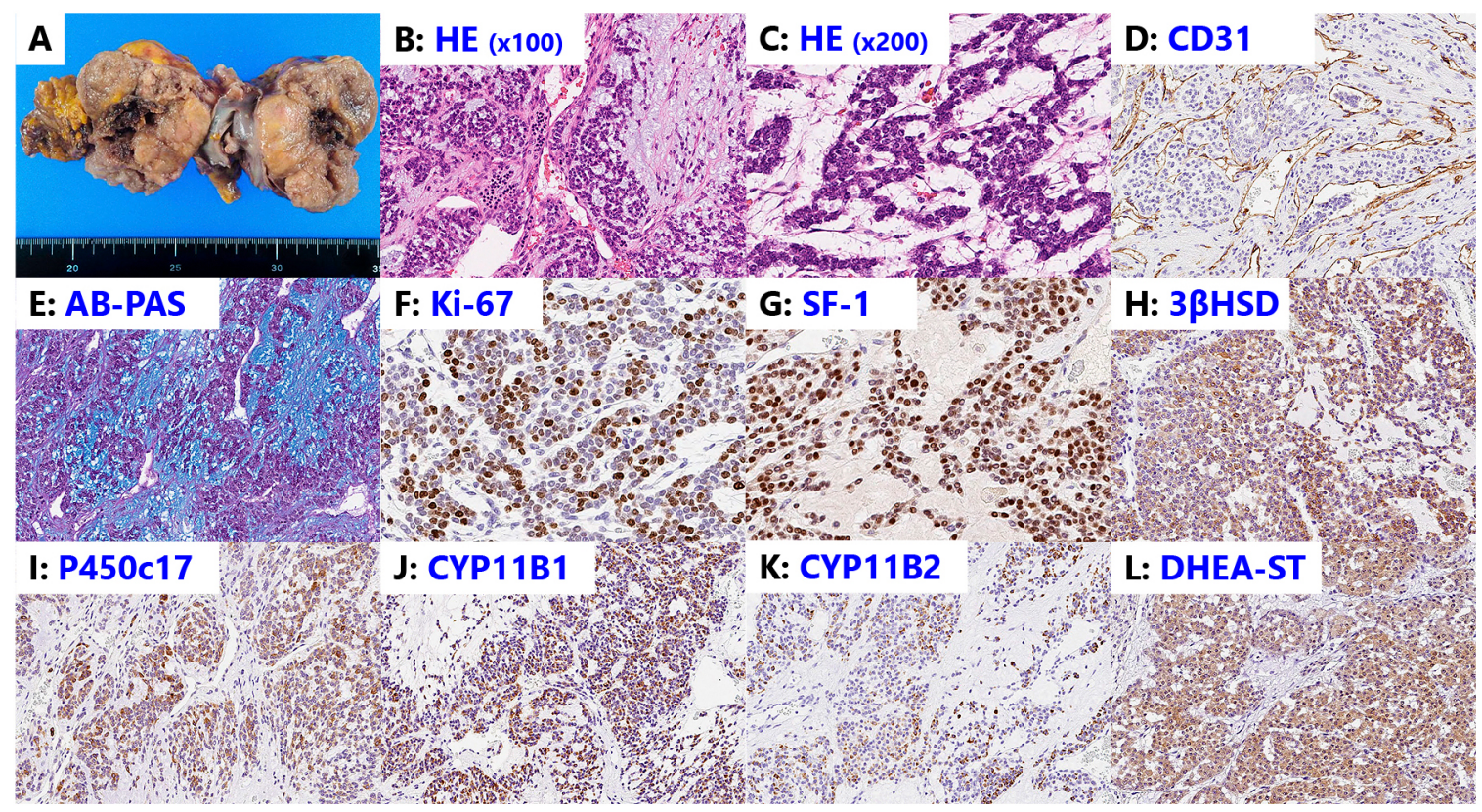

Fig. 3 Pathological findings. The adrenal tumor consists of a solitary dark-brown tumor with a thin fibrous capsule (A). Microscopically, the tumor presents a total Weiss score of 7 out of 9 points, including high nuclear grade, high mitotic rate, eosinophilic cytoplasm, diffuse architecture, venous invasion, sinusoidal invasion, and capsular invasion $(\mathrm{B}: \times 100, \mathrm{C}: \times 200$, hematoxylin and eosin staining, and D: $\times 100$, CD31 immunostaining). Alcian blue periodic acid-Schiff (AB-PAS) staining shows that the myxoid material is Alcian blue-positive (E). The Ki-67 labeling index is $21 \%$ in the hot spot (F). The tumor cells are immunohistochemically positive for steroidogenic factor 1 (SF-1) (G). In addition, they are positive for 3 $\beta$-hydroxysteroid dehydrogenase (3ßHSD) (H), 17 $\alpha$-hydroxylase (P450c17) (I), 11 $\beta$-hydroxylase (CYP11B1) (J), aldosterone synthase (CYP11B2) (K), and dehydroepiandrosterone sulfotransferase (DHEA-ST) (L). 
In the present case, the patient had a history of breast cancer and other MEN1-associated endocrine tumors. MEN1 is also related to the development of nonendocrine tumors, and female patients with MEN1 are reported to be at increased risk for breast cancer $[15,16]$. In cases of MEN1, the mechanisms of tumor formation may involve the loss of menin function in a tumor precursor cell [10]. Several cases have been reported in which patients with MEN1 developed multiple endocrine and non-endocrine tumors. Jeong et al. reported a case of MEN1 accompanied by breast cancer, thymic tumor, adrenal cortical adenoma, thyroid carcinoma, uterine leiomyoma, and lung hamartoma [17]. The patient in their study had a germline MEN1 gene mutation. In addition, Ohara et al. reported the case of a patient with MEN1 who developed both of adrenocortical carcinoma and lung adenocarcinoma [18]. In light of these previous cases and our own case, clinicians should be aware that both endocrine and non-endocrine tumors could possibly develop in patients with MEN1.

The myxoid variant of ACC was first reported in 1979 [19], and approximately 50 cases have been reported to date in the English-language literature to the best of our knowledge [9]. In the year 2017 World Health Organization classification, myxoid ACC was firstly recognized as the distinctive subtype of ACC [20]. Myxoid ACC is histologically characterized by the presence of abundant extracellular Alcian blue-positive myxoid material [21]. Tumor cells are generally arranged in cords, trabeculae, clusters, or pseudoglandular structures, often floating in pools or lakes of myxoid material [8]. These features could result in the difficulty of differential diagnosis, especially from metastatic adenocarcinoma to the adrenal gland [8, 20-22]. The majority of previously reported cases of myxoid ACC had clinical characteristics similar to those of conventional ACC, but Sung et al. indicated that the presence of myxoid histological features in ACC was indeed associated with more aggressive clinical behavior and poorer overall survival than conventional ACC [9], which may explain the relatively aggressive biological course of our patient.

Regarding the endocrinological features of ACC, approximately $60 \%$ of ACC patients present with an excess of adrenal steroid hormone, which most commonly manifests as Cushing's syndrome [2]. Aldosterone producing ACCs are relatively rare, accounting for approximately $13 \%$ of functioning ACCs, and manifest with hypertension with hypokalemia [23]. Our case was characterized by hypokalemia and hypertension on presentation, along with marked increases in plasma aldosterone and urine aldosterone, which was consistent with hyperaldosteronism.

In MEN1 patients, clinical and biochemical screening is recommended every 6-12 months, while radiological screening of the pancreas, adrenal glands, and pituitary gland using MRI or CT should be performed every 12 to 36 months [24, 25]. Our patient was followed-up within this recommended interval; however, the adrenal tumor became extremely enlarged especially in the last year. Our case may suggest the necessity of more close, regular follow-ups in patients with MEN1 accompanied by adrenal tumor, including imaging studies of adrenal tumor or observation of laboratory data associated with hyperaldosteronism and hypercortisolemia, considering the rapid growth of ACCs.

On unenhanced CT, ACCs rarely have an attenuation value of less than $10 \mathrm{HU}$, and the specificity of this threshold for the identification of benign adenomas is approximately $98 \%$ [26]. In our case, attenuation values of the tumor were approximately $30 \mathrm{HU}$ in the clinical course, which was consistent with an ACC. In addition, several case reports proposed that high signal intensity on T2-weighted imaging is one of the characteristics of myxoid adrenocortical tumors [27, 28], which was observed in our case. However, it is also a typical finding of other ACCs [26]; therefore, further reports are needed to determine imaging characteristics of myxoid ACCs.

In summary, we presented the first reported case of a myxoid variant of ACC in a patient with MEN1. The patient's clinical course was characterized by the development of multiple endocrine and non-endocrine tumors, as well as hyperaldosteronism. Despite surgery and mitotane therapy, the patient died of distant metastasis. The poor disease course and outcome detected in our present case are considered to be attributable to the presence of the myxoid histological variant. However, further studies are required to clarify the association between MEN1 and myxoid variants of ACC.

\section{Acknowledgements}

We are sincerely grateful to all of the clinical staff from the Department of General Medicine of our institution (Okayama University Graduate School of Medicine, Dentistry and Pharmaceutical Sciences) who contributed to the present work.

\section{Disclosure}

None of the authors have any potential conflicts of interest associated with this research. 


\section{References}

1. Fassnacht M, Kroiss M, Allolio B (2013) Update in adrenocortical carcinoma. J Clin Endocrinol Metab 98: 45514564.

2. Allolio B, Fassnacht M (2006) Clinical review: adrenocortical carcinoma: clinical update. J Clin Endocrinol Metab 91: 2027-2037.

3. Ng L, Libertino JM (2003) Adrenocortical carcinoma: diagnosis, evaluation and treatment. J Urol 169: 5-11.

4. de Krijger RR, Papathomas TG (2012) Adrenocortical neoplasia: evolving concepts in tumorigenesis with an emphasis on adrenal cortical carcinoma variants. Virchows Arch 460: 9-18.

5. Veytsman I, Nieman L, Fojo T (2009) Management of endocrine manifestations and the use of mitotane as a chemotherapeutic agent for adrenocortical carcinoma. J Clin Oncol 27: 4619-4629.

6. Balasubramaniam S, Fojo T (2010) Practical considerations in the evaluation and management of adrenocortical cancer. Semin Oncol 37: 619-626.

7. Duregon E, Cappellesso R, Maffeis V, Zaggia B, Ventura $\mathrm{L}$, et al. (2017) Validation of the prognostic role of the "Helsinki Score" in 225 cases of adrenocortical carcinoma. Hum Pathol 62: 1-7.

8. Weissferdt A, Phan A, Suster S, Moran CA (2013) Myxoid adrenocortical carcinoma: a clinicopathologic and immunohistochemical study of 7 cases, including 1 case with lipomatous metaplasia. Am J Clin Pathol 139: 780786.

9. Sung TY, Choi YM, Kim WG, Lee YM, Kim TY, et al. (2017) Myxoid and sarcomatoid variants of adrenocortical carcinoma: analysis of rare variants in single tertiary care center. J Korean Med Sci 32: 764-771.

10. Busygina V, Bale AE (2006) Multiple endocrine neoplasia type 1 (MEN1) as a cancer predisposition syndrome: clues into the mechanisms of MEN1-related carcinogenesis. Yale J Biol Med 79: 105-114.

11. Chandrasekharappa SC, Guru SC, Manickam P, Olufemi SE, Collins FS, et al. (1997) Positional cloning of the gene for multiple endocrine neoplasia-type 1. Science 276: 404-407.

12. Griniatsos JE, Dimitriou N, Zilos A, Sakellariou S, Evangelou K, et al. (2011) Bilateral adrenocortical carcinoma in a patient with multiple endocrine neoplasia type 1 (MEN1) and a novel mutation in the MEN1 gene. World $J$ Surg Oncol 9: 6.

13. Gatta-Cherifi B, Chabre O, Murat A, Niccoli P, CardotBauters C, et al. (2012) Adrenal involvement in MEN1. Analysis of 715 cases from the Groupe d'etude des Tumeurs Endocrines database. Eur $J$ Endocrinol 166: 269-279.

14. Mazzuco TL, Durand J, Chapman A, Crespigio J, Bourdeau I (2012) Genetic aspects of adrenocortical tumours and hyperplasias. Clin Endocrinol (Oxf) 77: 1-
10.

15. Sakurai A, Imai T, Kikumori T, Horiuchi K, Okamoto T, et al. (2013) Thymic neuroendocrine tumour in multiple endocrine neoplasia type 1: female patients are not rare exceptions. Clin Endocrinol (Oxf) 78: 248-254.

16. van Leeuwaarde RS, Dreijerink KM, Ausems MG, Beijers HJ, Dekkers OM, et al. (2017) MEN1-dependent breast cancer: indication for early screening? Results from the Dutch MEN1 Study Group. J Clin Endocrinol Metab 102: 2083-2090.

17. Jeong YJ, Oh HK, Bong JG (2014) Multiple endocrine neoplasia type 1 associated with breast cancer: a case report and review of the literature. Oncol Lett 8: 230-234.

18. Ohara N, Kaneko M, Ikeda M, Ishizaki F, Suzuki K, et al. (2017) Lung adenocarcinoma and adrenocortical carcinoma in a patient with multiple endocrine neoplasia type 1. Respir Med Case Rep 20: 77-81.

19. Tang CK, Harriman BB, Toker C (1979) Myxoid adrenal cortical carcinoma: a light and electron microscopic study. Arch Pathol Lab Med 103: 635-638.

20. Lloyd RV, Osamura RY, Klöppel G, Rosai J, Bosman FT, et al. (2017) WHO classification of tumours of endocrine organs. World Health Organization Classification of Tumours: 355 pages.

21. Erickson LA (2018) Challenges in surgical pathology of adrenocortical tumours. Histopathology 72: 82-96.

22. Miyoshi T, Otsuka F, Suzuki J, Inagaki K, Kano Y, et al. (2005) Abrupt enlargement of adrenal incidentaloma: a case of isolated adrenal metastasis. Endocr J 52: 785-788.

23. Abiven G, Coste J, Groussin L, Anract P, Tissier F, et al. (2006) Clinical and biological features in the prognosis of adrenocortical cancer: poor outcome of cortisol-secreting tumors in a series of 202 consecutive patients. J Clin Endocrinol Metab 91: 2650-2655.

24. Thakker RV, Newey PJ, Walls GV, Bilezikian J, Dralle H, et al. (2012) Clinical practice guidelines for multiple endocrine neoplasia type 1 (MEN1). J Clin Endocrinol Metab 97: 2990-3011.

25. Marini F, Giusti F, Tonelli F, Brandi ML (2017) Management impact: effects on quality of life and prognosis in MEN1. Endocr Relat Cancer 24: T227-T242.

26. Bharwani N, Rockall AG, Sahdev A, Gueorguiev M, Drake W, et al. (2011) Adrenocortical carcinoma: the range of appearances on CT and MRI. AJR Am J Roentgenol 196: W706-W714.

27. Fukuhara H, Bilim V, Ohtake H, Yahagi Y, Tomita Y (2013) A case of myxoid adrenocortical neoplasm: computed tomography and magnetic resonance imaging characteristics. Rare Tumors 5: e54.

28. Kim TU, Kim S, Lee JW, Lee NK, Ha HK, et al. (2014) Myxoid adrenocortical adenoma: magnetic resonance imaging and pathology correlation. Korean J Radiol 15: 245-249. 